\title{
Formation and stability of point defects in monolayer rhenium disulfide
}

\author{
S. Horzum,,${ }^{1,2,{ }^{*}}$ D. Çakır, ${ }^{1}$ J. Suh,${ }^{3}$ S. Tongay, ${ }^{3,4}$ Y.-S. Huang, ${ }^{5}$ C.-H. Ho, ${ }^{5}$ J. Wu,${ }^{3,4,6}$ H. Sahin,,${ }^{1, \dagger}$ and F. M. Peeters ${ }^{1, \ddagger}$ \\ ${ }^{1}$ Department of Physics, University of Antwerp, Groenenborgerlaan 171, B-2020 Antwerp, Belgium \\ ${ }^{2}$ Department of Engineering Physics, Faculty of Engineering, Ankara University, 06100 Ankara, Turkey \\ ${ }^{3}$ Department of Materials Science and Engineering, University of California, Berkeley, California 94720, USA \\ ${ }^{4}$ State Key Laboratory of Superlattices and Microstructures, Institute of Semiconductors, Chinese Academy of Sciences, P.O. Box 912 , \\ Beijing 100083, Peoples Republic of China \\ ${ }^{5}$ Department of Electronic Engineering, National Taiwan University of Science and Technology, Taipei 106, Taiwan \\ ${ }^{6}$ Materials Sciences Division, Lawrence Berkeley National Laboratory, Berkeley, California 94720, USA
}

(Received 6 February 2014; revised manuscript received 14 April 2014; published 28 April 2014)

\begin{abstract}
Recently, rhenium disulfide $\left(\operatorname{ReS}_{2}\right)$ monolayers were experimentally extracted by conventional mechanical exfoliation technique from as-grown $\mathrm{ReS}_{2}$ crystals. Unlike the well-known members of transition metal dichalcogenides (TMDs), $\mathrm{ReS}_{2}$ crystallizes in a stable distorted-1T structure and lacks an indirect to direct gap crossover. Here we present an experimental and theoretical study of the formation, energetics, and stability of the most prominent lattice defects in monolayer $\mathrm{ReS}_{2}$. Experimentally, irradiation with $3-\mathrm{MeV} \mathrm{He}^{+2}$ ions was used to break the strong covalent bonds in $\operatorname{ReS}_{2}$ flakes. Photoluminescence measurements showed that the luminescence from monolayers is mostly unchanged after highly energetic $\alpha$ particle irradiation. In order to understand the energetics of possible vacancies in $\mathrm{ReS}_{2}$ we performed systematic first-principles calculations. Our calculations revealed that the formation of a single sulfur vacancy has the lowest formation energy in both Re and $S$ rich conditions and a random distribution of such defects are energetically more preferable. Sulfur point defects do not result in any spin polarization whereas the creation of Re-containing point defects induce magnetization with a net magnetic moment of $1-3 \mu_{\mathrm{B}}$. Experimentally observed easy formation of sulfur vacancies is in good agreement with first-principles calculations.
\end{abstract}

DOI: 10.1103/PhysRevB.89.155433

PACS number(s): 71.20.Be, 75.70.Ak, 61.72.jd, 31.15.A-

\section{INTRODUCTION}

Graphene has become a material of great interest in the field of materials science since the successful synthesis of single layers $[1,2]$ and the theoretical investigations on its unusual properties $[3,4]$. Subsequent studies have revealed that graphenelike materials that possess a honeycomb lattice structure such as silicene [5,6], germanene [5], carbon nitride [7], III-V binary compounds [8], graphane [9,10], fluorographene [11,12], chlorographene [13], and transition metal dichalcogenides [14-17] (TMDs) are promising additions to graphene. The most popular member of single layer TMDs is molybdenum disulfide $\left(\mathrm{MoS}_{2}\right)$, but recent experimental studies have shown that the majority of dichalcogenides of molybdenum and tungsten can also be stable even in the single layer form [15-17]. Most of the TMDs have a graphenelike hexagonal crystal structure which is composed of layers of metal atoms $(M)$ sandwiched between layers of chalcogen atoms $(X)$ and these structures have $M X_{2}$ stoichiometry. Chalcogen layers can be stacked on top of each other in two different forms, namely an $H$ phase made of trigonal prismatic holes for metal atoms, and a $T$ phase that consists of staggered chalcogen layers forming octahedral holes for metals. As has been reported for $\mathrm{MoS}_{2}, \mathrm{MoSe}_{2}, \mathrm{WS}_{2}$, and $\mathrm{WSe}_{2}$, one of the characteristics of these materials is its indirect-to-direct band-gap transition that occurs when going from bulk to single layer [18-22]. Furthermore, for $\mathrm{MoSe}_{2}$

\footnotetext{
*seyda.horzumsahin@uantwerpen.be

†hasan.sahin@uantwerpen.be

†francois.peeters@uantwerpen.be
}

single layers, it was shown that applying biaxial tensile strain enables us to tune the electronic band structure and softens its phonon modes dramatically [23]. Very recently, we reported the accidentally degenerate nature of $A_{1 g}$ and $E_{2 g}$ modes and their substrate-sensitive behavior in $\mathrm{WSe}_{2}$ [24]. Chemical versatility and tunable electronic and vibrational properties of TMDs make these structures quite promising for nanoscale semiconductor device applications.

Despite the recent progress in experimental and theoretical investigation of other TMDs, studies on Rhenium disulfide $\left(\mathrm{ReS}_{2}\right)$ are rather sparse and mostly limited to its bulk structure. Early experiments demonstrated that bulk $\mathrm{ReS}_{2}$ has a lamellar structure and exhibits optical anisotropy in the van der Waals plane [25-27]. Differing from other TMDs bulk $\mathrm{ReS}_{2}$ is a semiconductor with a direct band gap of $1.47 \mathrm{eV}$ [28] that forms a distorted $\mathrm{CdCl}_{2}$-type structure. Motivated by our recent study [29] that reports the synthesis of $\mathrm{ReS}_{2}$ single layers and its electronic and vibrational properties, in this paper we focus on the formation and electronic properties of defects in these structures. Understanding the fundamental properties of $\mathrm{ReS}_{2}$, which is the newest member of the single layer TMD family, is expected to reveal new possibilities in this field of single atomic layered materials.

It is well known that during the growth and exfoliation of monolayer crystals formation of imperfections, e.g., point defects, grain boundaries, are unavoidable. These structural defects can have a significant influence on the electronic, optical, and mechanical properties of these ultrathin materials. $\mathrm{MoS}_{2}$ monolayers fabricated by using the chemical vapor deposition method have been shown to pose much lower carrier mobility, as compared to mechanically exfoliated monolayers, due to the presence of structural imperfections [30-32]. The 
performance of $\mathrm{ReS}_{2}$ in technological applications including optoelectronic and nanoelectronic devices critically depends on the quality of $\mathrm{ReS}_{2}$ layers. Therefore it is mandatory to study the formation and the stability of structural defects in this promising material.

The paper is organized as follows. We present the details of computational and experimental methodology in Sec. II. In Secs. III A and III B structural and electronic properties of single layer $\mathrm{ReS}_{2}$ are given, respectively. Experimental results on $\alpha$ irradiated $\mathrm{ReS}_{2}$ single layers are presented in Sec. IV. Energetics, formation, and magnetic properties of possible vacancy types in single layer $\operatorname{ReS}_{2}$ are discussed in Sec. V. A brief conclusion is given in Sec. VI.

\section{COMPUTATIONAL METHODOLOGY AND EXPERIMENTAL DETAILS}

First-principles calculations were carried out in the framework of density functional theory (DFT) as implemented in the Vienna $a b$ initio simulation package (VASP) [33,34]. The generalized gradient approximation (GGA) within PerdewBurke-Ernzerhof (PBE) formalism was employed for the exchange-correlation potential [35]. The projector augmented wave (PAW) method [36] and a plane-wave basis set with an energy cutoff of $500 \mathrm{eV}$ were used in the calculations. For geometry optimizations the Brillouin-zone integration was performed using a regular $3 \times 3 \times 1 k$ mesh within the Monkhorst-Pack scheme. In the electronic density of states calculations the Brillouin zone is sampled employing a $k$-point grid of $5 \times 5 \times 1$ [37]. The Gaussian smearing method was employed and the width of the smearing was chosen as $0.05 \mathrm{eV}$ in all relaxations. The convergence criterion of the self-consistent field calculations was set to $10^{-5} \mathrm{eV}$ for the total energy. To prevent spurious interaction between isolated $\mathrm{ReS}_{2}$ monolayers, a large vacuum spacing (at least $12 \AA$ ) was introduced. By using the conjugate gradient method, atomic positions and lattice constants were optimized until the atomic forces are less than $0.01 \mathrm{eV} / \AA$. Pressure on the supercells was decreased to values less than $1 \mathrm{kB}$. The defects were obtained by removing the relevant atoms from a $2 \times 2$ supercell containing 48 atoms. The convergence of defect formation energy was checked by varying the size of the supercell. Calculations with a $3 \times 3$ supercell, containing 96 atoms, show that the formation energy of defects are converged on the order of $0.01 \mathrm{eV}$, approaching the isolated defect limit.

Single and few layer $\mathrm{ReS}_{2}$ flakes were mechanically exfoliated from laboratory-grown bulk crystals onto 300-nm $\mathrm{SiO}_{2} / \mathrm{Si}$ wafer (MTI Corporation). The number of layers was determined by tapping-mode atomic force microscopy (AFM) scan and individual $\operatorname{ReS}_{2}$ monolayer displayed $\sim 0.7 \mathrm{~nm}$ thickness. Photoluminescence and Raman spectra were characterized using a Renishaw micro-Raman system with 488-nm laser. To study effects of defects, single and few layer flakes were irradiated using $3-\mathrm{MeV} \mathrm{He}^{2+}$ ions with a dose of $10^{14}$ $\mathrm{cm}^{-2}$.

\section{SINGLE LAYER RHENIUM DISULFIDE}

In Figs. 1(a) and 1(b), we display optical images of fewlayer and monolayer $\mathrm{ReS}_{2}$ flakes, respectively. Tapping mode
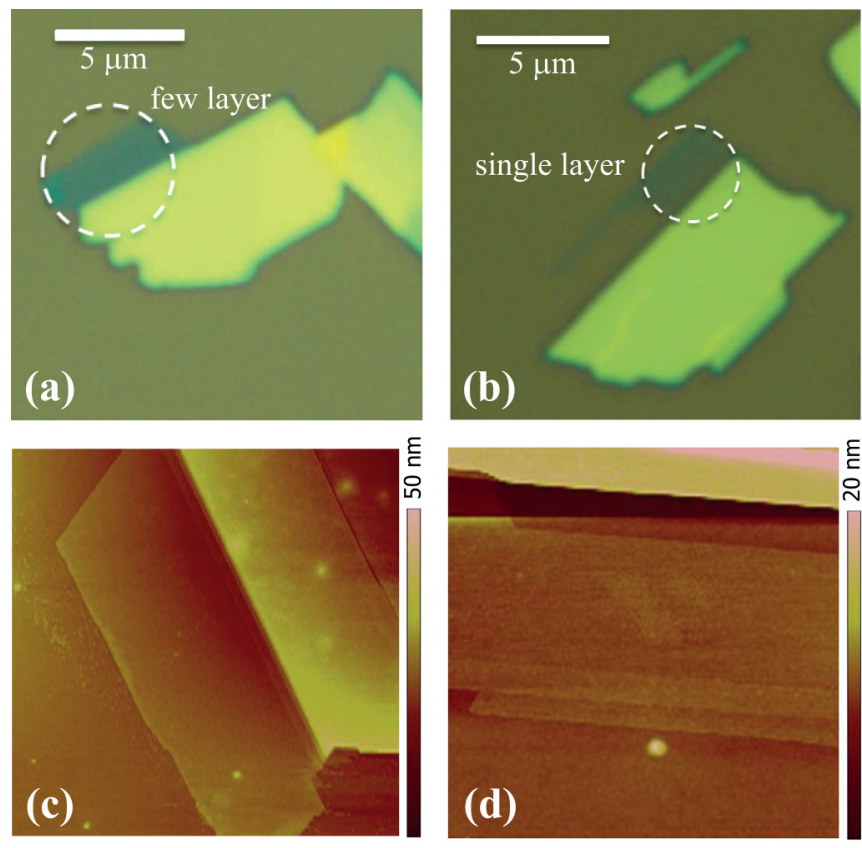

(e)

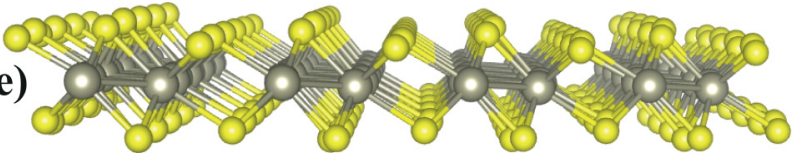

FIG. 1. (Color online) Optical image of (a) few-layer and (b) single layer $\mathrm{ReS}_{2}$ flakes exfoliated on 300-nm $\mathrm{SiO}_{2} / \mathrm{Si}$ substrates. Tapping mode AFM scan shows that the few-layer (c) and single layer (d) $\mathrm{ReS}_{2}$ flakes are smooth and highly crystalline. (e) Cross-sectional view of the atomic structure of a single layer $\mathrm{ReS}_{2}$.

AFM images in Figs. 1(c) and 1(d) show that the exfoliated flakes are atomically smooth and display rather sharp angles at the edges, highlighting the high crystallinity of the $\mathrm{ReS}_{2}$. Monolayer $\mathrm{ReS}_{2}$ flakes yield $\sim 0.7 \mathrm{~nm}$ thickness consistent with previously reported values [29]. Although $\mathrm{ReS}_{2}$ has the same chemical formula as other TMDs, theoretical analysis of the atomic structure given below reveals its distinctive properties.

Among the TMDs that have been reported to be stable individual layers of $\mathrm{MoS}_{2}, \mathrm{MoSe}_{2}, \mathrm{WS}_{2}$, and $\mathrm{WSe}_{2}$ have $1 \mathrm{H}$ structure in their ground state while dichalcogens of Ti, V, and Ta are in the $1 T$ phase. However, the atomic structure of $\mathrm{ReS}_{2}$ single layers have neither $H$ nor $T$ character.

Unlike other TMDs, $\operatorname{ReS}_{2}$ crystallizes in a distorted-1T $\left(1 T_{d}\right)$ structure with clustering of $\mathrm{Re}_{4}$ units forming parallel metal chains along the van der Waals plane. Spontaneous formation of such quasi-one-dimensional structures are the consequence of Jahn-Teller-type displacements of Re atoms. In Fig. 2(a), we present the optimized atomic structure of $\mathrm{ReS}_{2}$ in $1 H, 1 T$, and $1 T_{d}$ structures. Here PBE approximated lattice parameters $a$ for primitive cells are $3.31 \AA$ for $1 H, 3.10 \AA$ for $1 T$ and $6.51 \AA(b=6.40)$ for $1 T_{d}$. The formation of Re chains breaks the hexagonal symmetry and doubles the unit cell size. Hence the unit cell of single layer $\operatorname{ReS}_{2}$ in the $1 T_{d}$ phase is composed of four Re and eight $\mathrm{S}$ atoms. Here, since none of the chalcogen atoms in the unit cell are in the same plane, two-dimensional $\mathrm{ReS}_{2}$ can be thought of as a nine-layered 


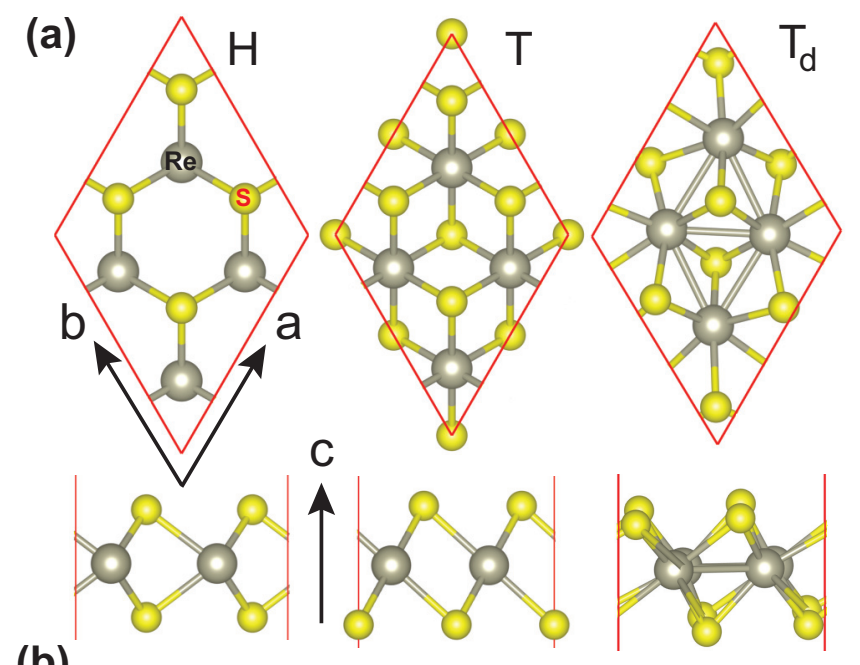

(b)

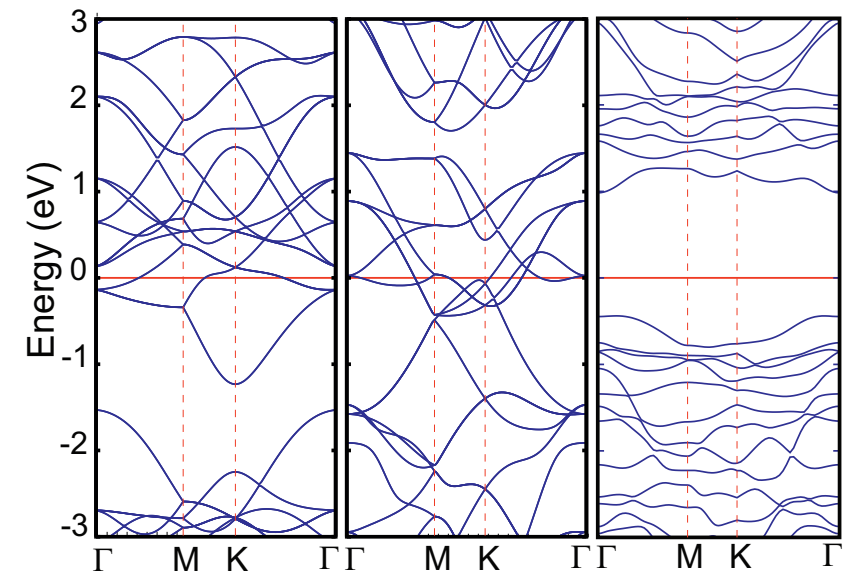

FIG. 2. (Color online) (a) Top and side view atomic structures of $1 \mathrm{H}, 1 \mathrm{~T}$, and distorted-1T $\left(1 T_{d}\right)$ phases of $\mathrm{ReS}_{2}$ monolayer. (b) Electronic band dispersions of the above three atomic structures.

structure in which the metal layer is sandwiched by eight layers of close-packed $\mathrm{S}$ atoms.

As we recently reported, periodic $1 H$ and $1 T$ structures of $\mathrm{ReS}_{2}$ can be obtained by total-energy optimization calculations, however from a calculation of the phonon spectrum we found that these phases of $\operatorname{ReS}_{2}$ cannot be stable in nature because the LA and ZA phonon modes that have imaginary frequencies [29]. It is also worthwhile to note that in addition to in- and out-of-plane anisotropy, $\operatorname{ReS}_{2}$ layers have also basal plane anisotropy along the $a$ and $b$ lattice vectors.

In Fig. 2(b), we present the electronic band dispersion for single layer $\mathrm{ReS}_{2}$. While $1 H$ and $1 T$ phases exhibit metallic behavior, the $1 T_{d}$ structure is a semiconductor with a calculated band gap of $1.43 \mathrm{eV}$. Photoluminescence measurements have shown that the single layer $\mathrm{ReS}_{2}$ is a direct-gap semiconductor with a gap of $1.55 \mathrm{eV}$ and does not exhibit a direct-to-indirect gap transition when going from single layer to bulk [29], which makes this material attractive for optoelectronic applications. Our DFT calculations show that the valence band at the close proximity of the $\Gamma$ point along the $\Gamma-M$ direction is quite flat. The direct (at $\Gamma$ ) and indirect (at $\Gamma K \longrightarrow \Gamma$ and $\Gamma M \longrightarrow \Gamma$ ) band gaps are almost degenerate with an energy difference of $8 \mathrm{meV}$. However, this artificial indirect behavior disappears and the band gap becomes direct when spin-orbit interactions are taken into account. From an experimental point of view, the distorted nature of basal plane is expected to result in anisotropic electronic, optical, and mechanical properties of the $\mathrm{ReS}_{2}$ compound.

\section{CREATING DEFECTS BY $\alpha$-PARTICLE IRRADIATION}

To study the effect of sulfur vacancies, we irradiated $\mathrm{ReS}_{2}$ flakes with $3-\mathrm{MeV} \mathrm{He} \mathrm{He}^{+2}$ ions (with an ion fluence of $1 \times 10^{14} \mathrm{~cm}^{-2}$ ). Here, $\mathrm{He}^{+2}$ ions heavily interact with the $\mathrm{ReS}_{2}$ flakes by multiple collisions and this technique is expected to create various vacancies as studied in other monolayer TMDs [38]. We present photoluminescence (PL) measurements performed on pristine and irradiated $\mathrm{ReS}_{2}$ flakes as shown in Fig. 3. Prior to irradiation, the $\mathrm{ReS}_{2}$ flakes display a broad PL signal located around $1.50-1.65 \mathrm{eV}$.

In Fig. 3(a) we present the PL spectrum of pristine and irradiated samples measured at $300 \mathrm{~K}$. After the bombardment, overall PL peak shape remains the same but the intensity is slightly reduced. We attribute such reduction in the PL signal to an increase in nonradiative recombination processes associated with the created defect centers. Increased defect density is known to reduce the quantum yield by reducing the radiative recombination probability through interaction at the defect sites. However, we also note that conclusions about PL efficiency are speculative as the measurement technique (photoluminescence) cannot comment on the absolute PL efficiency.

Here, we also note that the creation of defects in twodimensional materials such as $\mathrm{ReS}_{2}$ is expected to have rather
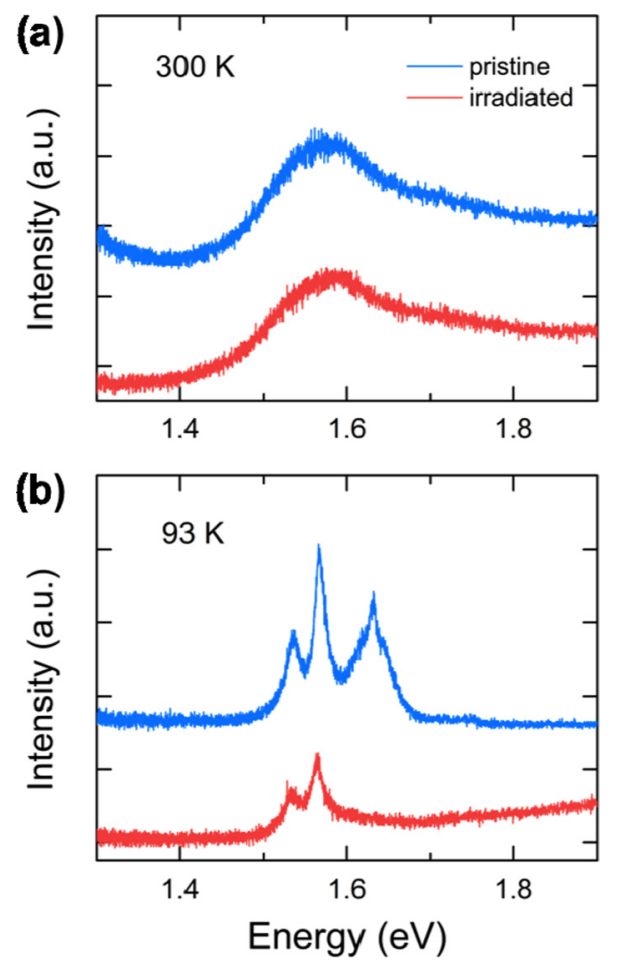

FIG. 3. (Color online) PL spectrum taken on monolayer $\mathrm{ReS}_{2}$ flakes (a) at $300 \mathrm{~K}$ and (b) $93 \mathrm{~K}$ before (blue) and after (red) $\alpha$ particle irradiation. 

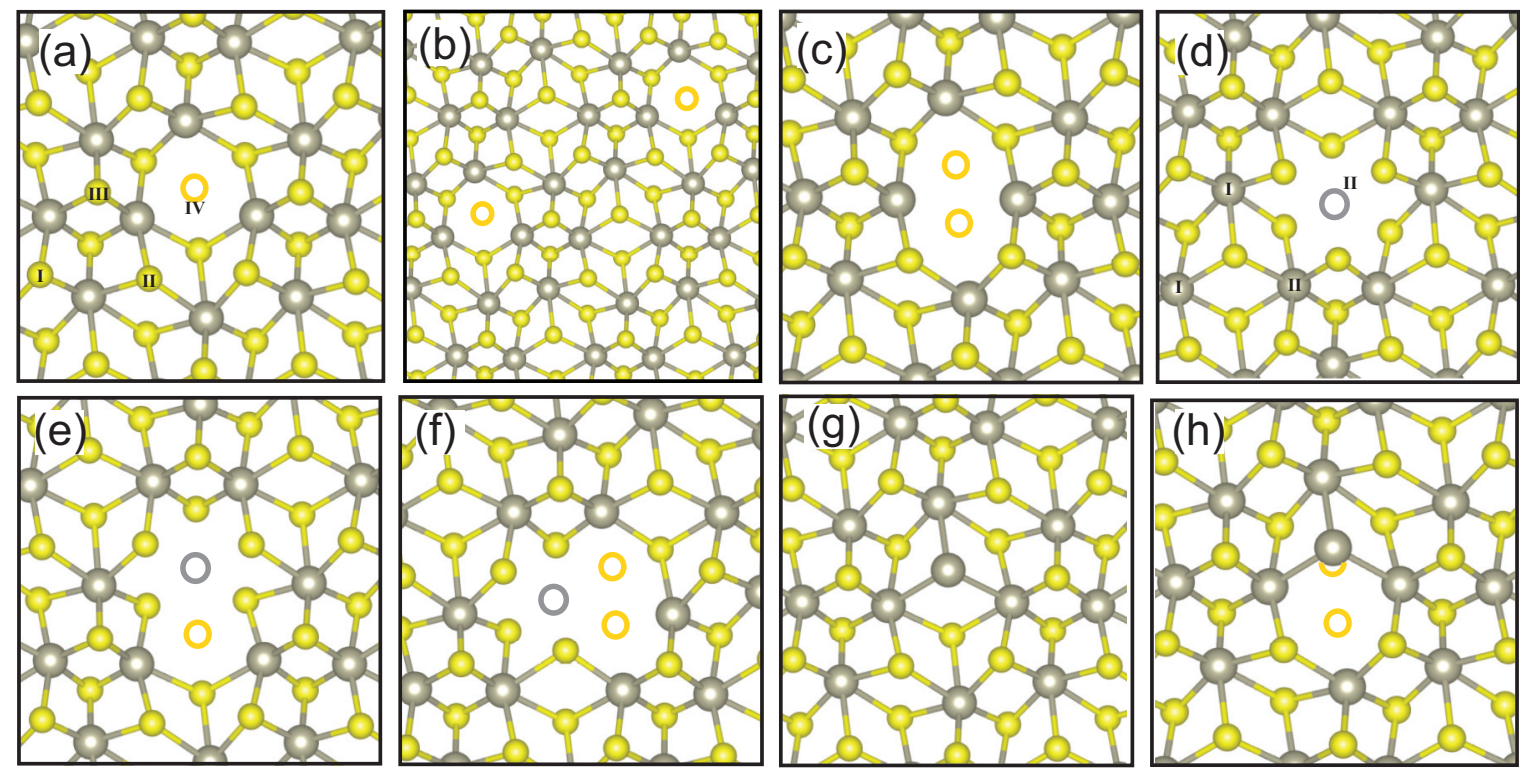

FIG. 4. (Color online) Atomic structure of (a) monosulfur vacancy $\mathrm{V}_{S}$ (b) two monosulfur vacancies $\mathrm{V}_{S+S}$ (c) disulfur vacancy $\mathrm{V}_{2 S}$ (d) Re vacancy $\mathrm{V}_{R e}$ (e) $R e S$ vacancy $\mathrm{V}_{R e S}$ (f) $R_{e} S_{2}$ vacancy $\mathrm{V}_{R e S_{2}}$ (g) single $\mathrm{S}$ atom substituted by a $\mathrm{Re}$ atom $\mathrm{S}_{S \rightarrow R e}$, and (h) two $\mathrm{S}$ atoms substituted by a $\operatorname{Re}$ atom $\mathrm{S}_{2 S \rightarrow R e}$. Missing $\operatorname{Re}(\mathrm{S})$ atoms are shown by empty gray (yellow) circles. In (a) and (d), the numbered atoms indicate the possible inequivalent sites for creating $\mathrm{S}$ and Re vacancies, respectively.

strong effects on the optical properties as the excitons are strictly localized in two-dimensions and strongly interact with the created imperfections/defects. On the contrary, our micro-PL measurements show that the PL spectrum is largely unchanged. Previously, the presence of defects have been studied in $\mathrm{MoS}_{2}, \mathrm{MoSe}_{2}, \mathrm{WS}_{2}$, and $\mathrm{WSe}_{2}$ [38]. In the latter work, the authors have reported that the presence of defects did not cause any significant changes in the PL spectrum at room temperature but the bound exciton peak $\left(X_{B}\right)$ became observable below the band-gap light emission peak only at $77 \mathrm{~K}$.

Next, we discuss our photoluminescence measurements taken at $93 \mathrm{~K}$. Figure 7(b) shows the PL spectrum taken on monolayer $\mathrm{ReS}_{2}$ prior to particle bombardment where the spectrum consists of three sharp emission lines that are roughly separated by $50-70 \mathrm{meV}$. Indeed, similar PL emission lines have been measured on $\mathrm{ReS}_{2}$ bulk crystals and they have been attributed to various interband transitions occurring in different polarization directions within the inplane crystal which is strongly anisotropic due to strong Re-Re interaction/dimerization [39]. Similar to the referenced work, distinct PL peak emissions might be related to various interband transitions. Interestingly, after irradiation (defect creation), the highest energy peak disappears. The latter effect might be related to reduction of the in-plane crystal anisotropy and blocking one of the radiative recombination processes.

\section{ENERGETICS OF VARIOUS POINT DEFECTS}

Existence of lattice imperfections in two-dimensional graphenelike structures not only creates localized electronic and excitonic states, but can also lead to additional magnetic properties. Depending on the synthesis and transfer procedure various types of defects are quite likely to be formed. Recent studies have shown that controllable formation of vacancies in monolayer transition metal dichalcogenides can be realized experimentally by $\alpha$-particle irradiation and thermal annealing. In general, atomic vacancies largely affect the electronic, magnetic, and structural properties, and therefore, it is essential to investigate the characteristic properties of different point defects.

\section{A. Structural properties and energetics}

In this work we will consider the most commonly observed point defects in TMDs such as monosulfur vacancy $\left(\mathrm{V}_{S}\right)$, disulfur vacancy $\left(\mathrm{V}_{2 S}\right)$, two monosulfur vacancies on different parts of the $\operatorname{ReS}_{2}$ monolayer $\left(\mathrm{V}_{S+S}\right)$, Re vacancy $\left(\mathrm{V}_{R e}\right)$, vacancy complex of Re and $\mathrm{S}$ vacancies $\left(\mathrm{V}_{R e S}\right)$, vacancy complex of Re and disulfur vacancy $\left(\mathrm{V}_{R e S_{2}}\right)$, antisite defects where a Re atom is substituting a $\mathrm{S}\left(\mathrm{S}_{S \rightarrow R e}\right)$ and two neighboring $\mathrm{S}$ atoms $\left(\mathrm{S}_{2 S \rightarrow R e}\right)$. Pertinent atomic structures obtained after full geometry optimization are shown in Figs. 4(a)-4(h). We investigate the relative stability of those point defects by calculating the formation energy using the following expression:

$$
E_{f}=E_{\mathrm{tot}}(\mathrm{def})+\sum_{i} N \mu_{i}-E_{\mathrm{tot}}(\text { pristine })
$$

where $E_{\mathrm{tot}}(\mathrm{def})$ and $E_{\mathrm{tot}}$ (pristine) are the total energies of the $\mathrm{ReS}_{2}$ supercell with and without defects, $\mu_{i}$ and $N$ are the chemical potential and the number of removed or added atoms. Normally the choice of $\mu_{i}$ is determined by experimental conditions. The defect formation energies as a function of the $\mathrm{S}$ chemical potential over a wide range of experimental conditions from $\mathrm{S}$ poor to $\mathrm{S}$ rich are plotted in Fig. 5. At equilibrium, we have

$$
2 \mu_{S}+\mu_{R e}=\mu_{R e S_{2}}=2 \mu_{S}^{\text {bulk }}+\mu_{R e}^{\text {bulk }}-\Delta H_{f},
$$




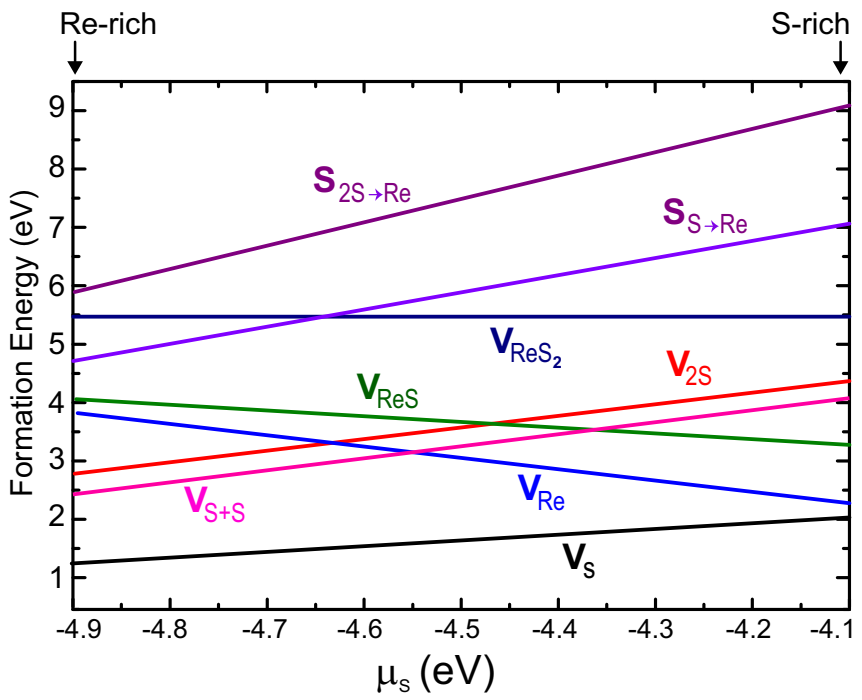

FIG. 5. (Color online) Formation energies of vacancy $(\mathbf{V})$ and substitutional (S) point defects as a function of the $\mathrm{S}$ chemical potential.

where $\Delta H_{f}$ is the heat of formation and is found to be $-1.75 \mathrm{eV}$. In Fig. 5, left and right boundaries correspond to two different experimental conditions. At the left boundary (corresponding to S-poor or Re-rich environment), bulk Re is used as a reservoir to determine the chemical potential of S using $\mu_{S}=\left(\mu_{R e S_{2}}-\mu_{R e}\right) / 2$, where $\mu_{R e S_{2}}$ is set to the total energy of pristine $\mathrm{ReS}_{2}$. Similarly, at the right boundary (corresponding to Re-poor or S-rich conditions), bulk S was chosen as the reservoir and the chemical potential of $\mathrm{Re}$ is calculated by using $\mu_{R e}=\mu_{R e S_{2}}-2 \mu_{S}$. Again, $\mu_{R e S_{2}}$ is the total energy of a clean $\operatorname{ReS}_{2}$ monolayer.

Due to the distorted lattice structure of $\mathrm{ReS}_{2}$, the formation of all types of point defects considered in this work are site dependent. The values presented in Table I are for those defect sites with the lowest formation energy. As labeled in Fig. 4(a) one can consider four different sites to create a $S$ vacancy. We calculated the S-vacancy formation energies at I, II, III, and IV sites as 2.06 (2.93), 1.63 (2.50), 2.36 (3.23), and 1.16 (2.03) eV at the $\operatorname{Re}(\mathrm{S})$-rich condition, respectively. The

TABLE I. Calculated values for different point defects in $\mathrm{ReS}_{2}$; lattice constants $|a|$ and $|b|$ (in a pristine $3 \times 3 \operatorname{ReS}_{2}$ monolayer $|a|=$ $19.54 \AA$ and $|b|=19.21 \AA$ ), total magnetic moment of the system $\left(\mu_{\mathrm{Tot}}\right)$, formation energy for the Re-rich case $\left(E_{f, R e}\right)$, formation energy for the $S$-rich case $\left(E_{f, S}\right)$, and energy band gap $E_{\text {gap }}$.

\begin{tabular}{lccccc}
\hline \hline Defect & $\begin{array}{c}|a|,|b| \\
(\AA)\end{array}$ & $\begin{array}{c}\mu_{\text {Tot }} \\
\left(\mu_{\mathrm{B}}\right)\end{array}$ & $\begin{array}{c}E_{f, R e} \\
(\mathrm{eV})\end{array}$ & $\begin{array}{c}E_{f, S} \\
(\mathrm{eV})\end{array}$ & $\begin{array}{c}E_{\text {gap }} \\
(\mathrm{eV})\end{array}$ \\
\hline $\mathbf{V}_{\boldsymbol{S}}$ & $19.50,19.17$ & 0 & 1.16 & 2.03 & 1.27 \\
$\mathbf{V}_{S+S}$ & $19.46,19.11$ & 0 & 2.36 & 4.10 & 1.26 \\
$\mathbf{V}_{\mathbf{2 S}}$ & $19.45,19.11$ & 0 & 2.63 & 4.37 & 1.08 \\
$\mathbf{V}_{\boldsymbol{R e}}$ & $19.55,19.22$ & 1 & 4.02 & 2.27 & 0.35 \\
$\mathbf{V}_{\boldsymbol{R e S}}$ & $19.57,19.27$ & 1 & 4.15 & 3.27 & 0.25 \\
$\mathbf{V}_{\boldsymbol{R e S} S_{\mathbf{2}}}$ & $19.55,19.16$ & 3 & 5.47 & 5.47 & 0.30 \\
$\mathbf{S}_{S \rightarrow \boldsymbol{R} e}$ & $19.57,19.23$ & 3 & 4.45 & 7.06 & 1.03 \\
$\mathbf{S}_{\mathbf{2 S} \rightarrow \boldsymbol{R} \boldsymbol{e}}$ & $19.53,19.18$ & 3 & 5.61 & 9.09 & 0.82 \\
\hline \hline
\end{tabular}

calculated formation energies reveal that an $\mathrm{S}$ vacancy tends to form between ReS chains, depicted in Fig. 4(a). While the formation energy of $\mathrm{V}_{S}$ is $1.16 \mathrm{eV}$ at the Re-rich condition, it increases to $2.03 \mathrm{eV}$ at the $\mathrm{S}$-rich condition, meaning that the possibility of the formation of an $\mathrm{S}$ vacancy decreases when one increases the amount of $\mathrm{S}$ atoms that are used to fabricate $\mathrm{ReS}_{2}$ monolayer.

Next we investigate the formation of a second $S$ vacancy on the $\mathrm{ReS}_{2}$ monolayer having already a single $\mathrm{S}$ vacancy. Comparison of the formation energies of $\mathrm{V}_{S+S}$ and $\mathrm{V}_{S}$ defects clearly shows that the repulsive interaction between two monosulfur vacancies is small with an interaction energy of $40 \mathrm{meV}$ including relaxation effects as well. In addition we calculate the formation energy of two $\mathrm{S}$ vacancies $\left(\mathrm{V}_{2 S}\right)$ which is created by removing two $\mathrm{S}$ atoms from the same sublattice. It appears that the formation of well-separated two $S$ vacancies (i.e., $\mathrm{V}_{S+S}$ ) is more favorable than that of $\mathrm{V}_{2 S}$, suggesting that two $\mathrm{V}_{S}$ do not have the tendency to combine. In other words, unlike graphene in which $\mathrm{C}$ vacancies favor to form vacancy complexes rather than staying isolated, a random distribution of $\mathrm{V}_{S}$ defects is energetically more favorable in a $\mathrm{ReS}_{2}$ monolayer.

As listed in Table I, Re including vacancies have different characteristics. In addition to their quite high formation energies, they result in a spin polarized ground state. Among the Re related defects, $\mathrm{V}_{R e}$ has the lowest formation energy. Possible Re sites (I and II) for creating a Re vacancy are shown in Fig. 4(d). The formation energy of a Re vacancy at site II is $0.4 \mathrm{eV}$ lower as compared to site I. It is seen from Fig. 4 that the creation of a $\mathrm{Re}$ vacancy $\left(\mathrm{V}_{R e}\right)$ leaves six $\mathrm{S}$ radicals which are hardly bound, whereas the removal of a $S$ atom results in three Re radicals having additional interaction with each other, lowering the formation energy.

Analysis of optimized lattice constants summarized in Table I shows that S-containing defects results in a small but noticeable contraction of the lattice, whereas Re-containing defects work adversely. Apparently, among the various point defect types single $\mathrm{S}$ vacancy $\mathrm{V}_{S}$ has the lowest formation energy and therefore the creation of such defects is most likely. However, at thermal equilibrium, the formation of $\mathrm{S}$ and $\mathrm{Re}$ containing vacancy complexes such as $\mathrm{V}_{R e S_{2}}$ with formation energy of 4.0-5.5 eV is quite difficult.

It is now useful to compare the formation energies of Re and $S$ vacancies in $\operatorname{ReS}_{2}$ with that of Mo and S vacancies in $\mathrm{MoS}_{2}$. At $\mathrm{Re}$ - and Mo-rich conditions, the formation energies for $\mathrm{Re}$ and Mo vacancies are 4.02 and $7.18 \mathrm{eV}$, respectively. Here, the chemical potential of Mo atoms is set to the total energy of the bulk phase of Mo. Similarly, for S vacancies at the S-rich condition, the formation energies are calculated as $2.03 \mathrm{eV}$ for $\mathrm{ReS}_{2}$ and $2.65 \mathrm{eV}$ for $\mathrm{MoS}_{2}$ monolayers. It appears that, due to the relatively soft covalent bonding in distorted-1T structure of $\mathrm{ReS}_{2}$, the formation of point defects in a $\mathrm{ReS}_{2}$ monolayer is more likely as compared to the $\mathrm{MoS}_{2}$ monolayer case.

\section{B. Electronic properties}

As shown in Fig. 6 and listed in Table I, the presence of point defects can lead to modifications in the electronic and the magnetic properties of the $\operatorname{ReS}_{2}$ monolayer. In the case of $\mathrm{S}$ vacancy, it is expected that three Re atoms easily saturate their 


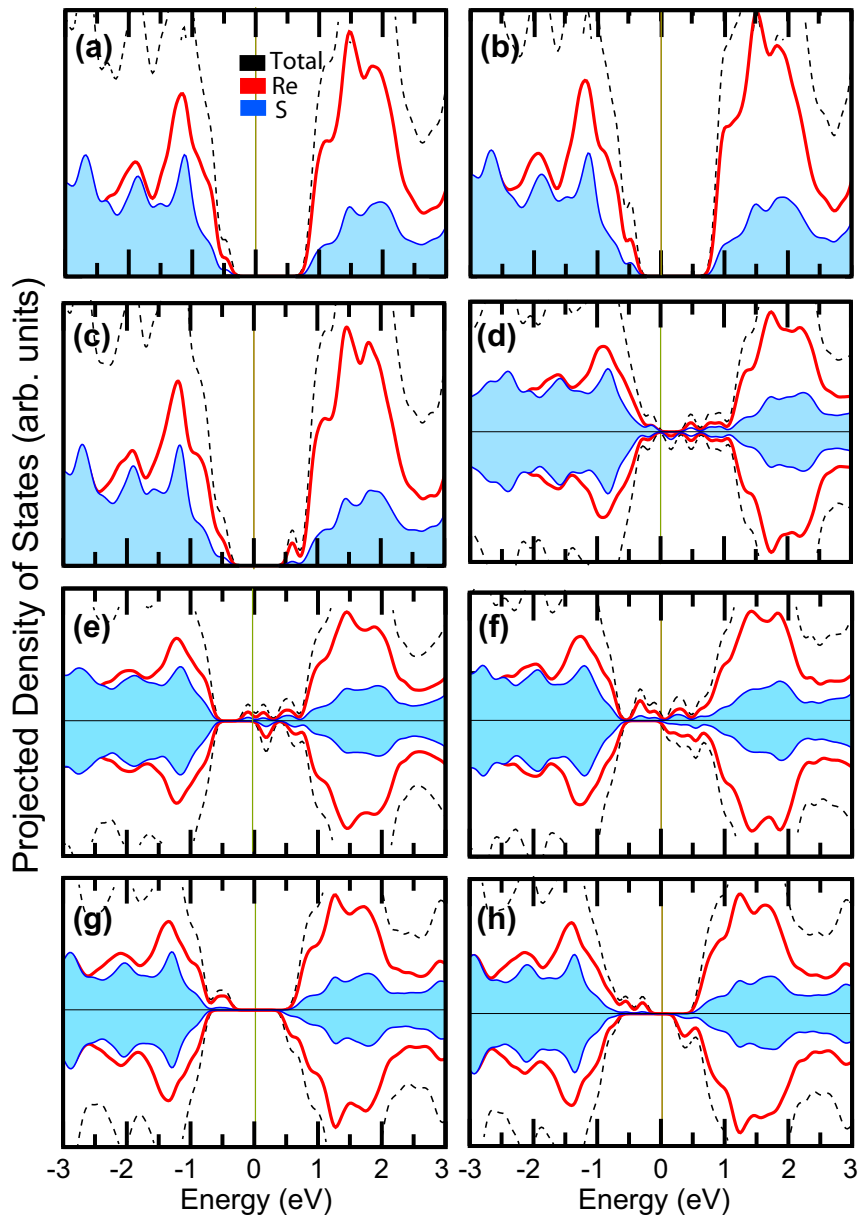

FIG. 6. (Color online) Density of states of (a) $\mathrm{V}_{S}$, (b) $\mathrm{V}_{S+S}$, (c) $\mathrm{V}_{2 S}$, (d) $\mathrm{V}_{R e}$, (e) $\mathrm{V}_{R e S}$, (f) $\mathrm{V}_{R e S_{2}}$, (g) $\mathrm{S}_{S \rightarrow R e}$, and (h) $\mathrm{S}_{2 S \rightarrow R e}$. For structures with spin polarization (d)-(h) in their ground state partial density of states of spin up and down states are presented separately.

unpaired electrons by forming additional bonds, which confirms our finding that the $\mathrm{ReS}_{2}$ monolayer with $\mathrm{S}$ vacancies has zero magnetic moment. A similar behavior can be seen even for $\mathrm{V}_{S+S}$ and $\mathrm{V}_{2 S}$ point defects. Here it appears that the DOS of $\mathrm{V}_{2 S}$ defects is slightly different from those of other $\mathrm{S}$ vacancy defects. Interestingly, the band gap of clean $\mathrm{ReS}_{2}$ does not change much upon the creation of a $S$ vacancy. The formation of the $\mathrm{S}$ vacancy reduces the band gap from $1.43 \mathrm{eV}$, the gap of clean $\mathrm{ReS}_{2}$, to $1.27 \mathrm{eV}$. Since two $\mathrm{S}$ atoms are removed, $\mathrm{V}_{2 S}$ reduces the band gap of clean $\mathrm{ReS}_{2}$ further to $1.08 \mathrm{eV}$. However, when we create two $S$ vacancies further away from each other on $\mathrm{ReS}_{2}$, the gap of the resultant monolayer is $1.25 \mathrm{eV}$ being very close to that of the single $\mathrm{S}$ vacancy.

Among the point defects in which Re atoms are involved, the ground state is found to be magnetic. Once a Re vacancy is formed, magnetization with a magnetic moment of $\mu=1 \mu_{\mathrm{B}}$ appears. The energy difference between spin polarized and spin unpolarized states is calculated to be $57 \mathrm{meV}$, indicating the stability of the magnetic state at fairly high temperatures. However, in single layer $\mathrm{MoS}_{2}$, such energy difference was found to be only $5 \mathrm{meV}$ [40]. Magnetization density (i.e., $\Delta \rho_{\uparrow, \downarrow}=\rho_{\uparrow}-\rho_{\downarrow}$ ) for the Re vacancy is depicted in Fig. 7. As seen from Fig. 7 the main contribution to the magnetization

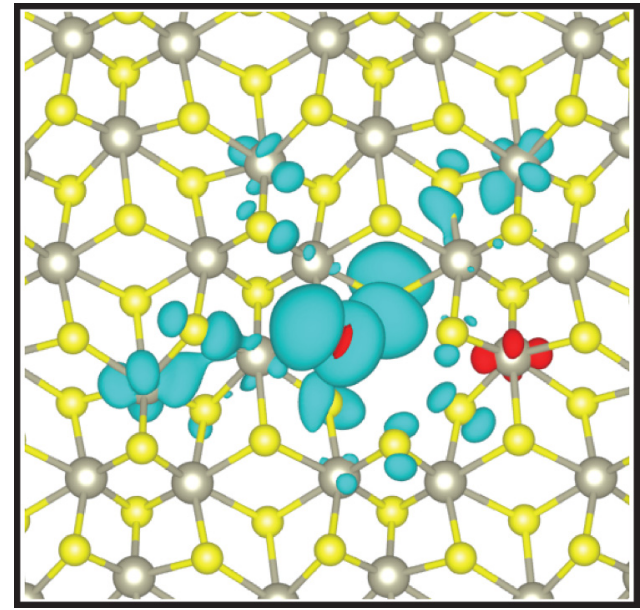

FIG. 7. (Color online) The spin resolved charge density for Re vacancy (isosurface value $=0.001 \mathrm{e} / \AA^{3}$ ). Blue (red) colors represents spin up (down) electrons.

comes from $p$ orbitals of two neighboring $\mathrm{S}$ atoms within the vacancy region. Although GGA functionals generally give satisfactory results for transition metals, the role of the local Coulomb interactions in partially filled $d$ orbitals needs further attention. We also checked whether or not Re vacancy induced magnetization is sensitive to the computational methodology used in the calculations. In these calculations, the Hubbard $U$ parameter for $d$ orbitals of Re and $p$ orbitals of $\mathrm{S}$ was 2 and $1 \mathrm{eV}$, respectively. In this $\mathrm{GGA}+U$ settings, the energy difference between spin polarized and unpolarized states becomes $0.1 \mathrm{eV}$. Our results show that the defect-induced electronic and magnetic structure of $\operatorname{ReS}_{2}$ is the same in the framework of both GGA and GGA $+U$. In contrast to $\mathrm{V}_{S}$, $\mathrm{V}_{S+S}$, and $\mathrm{V}_{2 S}$, Re-related defects give rise to several gap states and consequently reduces the effective band gap of pristine $\mathrm{ReS}_{2}$ significantly. For instance, in the case of $\mathrm{V}_{R e}$, the band gap of the defected system becomes only $0.35 \mathrm{eV}$.

\section{CONCLUSION}

In this work, first we experimentally investigated what kind of defects can be formed in $\mathrm{ReS}_{2}$ monolayers upon irradiation with $3 \mathrm{MeV} \mathrm{He}^{+2}$ ions. Interestingly, our photoluminescence measurements showed that ion bombardment does not result in a significant modification of the electronic structure of single layers. To explain the experimental findings we systematically investigated the formation and energetics of different point defects $\left(\mathrm{V}_{S}, \mathrm{~V}_{S+S}, \mathrm{~V}_{2 S}, \mathrm{~V}_{R e}, \mathrm{~V}_{R e S}, \mathrm{~V}_{R e S_{2}}, \mathrm{~S}_{S \rightarrow R e}\right.$, and $\left.\mathrm{S}_{2 S \rightarrow R e}\right)$ in a $\mathrm{ReS}_{2}$ monolayer. It was found that the formation of the defects does not destroy the semiconductor nature of $\mathrm{ReS}_{2}$. Re-containing point defects lead to spin polarized ground states with localized magnetic moments of $1-3 \mu_{\mathrm{B}}$, while S-containing defects result in a nonmagnetic semiconducting ground state. It was also found theoretically that the formation of sulfur vacancies are the most likely in monolayers of $\mathrm{ReS}_{2}$ while a large amount of energy is required for the creation of Re-containing defects. Experimentally observed easier formation of sulfur vacancies that result in no significant change in the electronic structure of single layer $\mathrm{ReS}_{2}$ agrees well with our theoretical results. 


\section{ACKNOWLEDGMENTS}

This work was supported by the Flemish Science Foundation (FWO-Vl), the bilateral project FWO-TUBITAK, and the Methusalem Foundation of the Flemish government.
Computational resources were provided by TUBITAK ULAKBIM, High Performance and Grid Computing Center (TR-Grid e-Infrastructure). H.S. was supported by a FWO Pegasus Long Marie Curie Fellowship. D.C. was supported by a FWO Pegasus-short Marie Curie Fellowship.
[1] K. S. Novoselov, A. K. Geim, S. V. Morozov, D. Jiang, S. C. Dubonos, I. V. Grigorieva, and A. A. Firsov, Science 306, 666 (2004).

[2] A. K. Geim and K. S. Novoselov, Nat. Mater. 6, 183 (2007).

[3] A. H. C. Neto, F. Guinea, N. M. R. Peres, K. S. Novoselov, and A. K. Geim, Rev. Mod. Phys. 81, 109 (2009).

[4] S. D. Sarma, S. Adam, E. H. Hwang, and E. Rossi, Rev. Mod. Phys. 83, 407 (2011).

[5] S. Cahangirov, M. Topsakal, E. Akturk, H. Sahin, and S. Ciraci, Phys. Rev. Lett. 102, 236804 (2009).

[6] P. Vogt, P. De Padova, C. Quaresima, J. Avila, E. Frantzeskakis, M. C. Asensio, A. Resta, B. Ealet, and G. LeLay, Phys. Rev. Lett. 108, 155501 (2012).

[7] A. Y. Liu and M. L. Cohen, Science 245, 841 (1989).

[8] H. Sahin, S. Cahangirov, M. Topsakal, E. Bekaroglu, E. Akturk, R. T. Senger, and S. Ciraci, Phys. Rev. B 80, 155453 (2009).

[9] J. O. Sofo, A. S. Chaudhari, and G. D. Barber, Phys. Rev. B 75, 153401 (2007).

[10] H. Sahin, C. Ataca, and S. Ciraci, Appl. Phys. Lett. 95, 222510 (2009).

[11] H. Sahin, M. Topsakal, and S. Ciraci, Phys. Rev. B 83, 115432 (2011).

[12] O. Leenaerts, H. Peelaers, A. D. Hernandez-Nieves, B. Partoens, and F. M. Peeters, Phys. Rev. B 82, 195436 (2010).

[13] H. Sahin and S. Ciraci, J. Phys. Chem. C 116, 24075 (2012).

[14] A. H. C. Neto and K. Novoselov, Rep. Prog. Phys. 74, 082501 (2011).

[15] C. Ataca, H. Sahin, and S. Ciraci, J. Phys. Chem. C 116, 8983 (2012).

[16] K. S. Novoselov, D. Jiang, F. Schedin, T. J. Booth, V. V. Khotkevich, S. V. Morozov, and A. K. Geim, Proc. Natl. Acad. Sci. USA 102, 10451 (2005).

[17] J. N. Coleman et al., Science 331, 568 (2011).

[18] R. A. Gordon, D. Yang, E. D. Crozier, D. T. Jiang, and R. F. Frindt, Phys. Rev. B 65, 125407 (2002).

[19] Q. H. Wang, K. Kalantar-Zadeh, A. Kis, J. N. Coleman, and M. S. Strano, Nat. Nanotechnol. 7, 699 (2012).

[20] K. F. Mak, C. Lee, J. Hone, J. Shan, and T. F. Heinz, Phys. Rev. Lett. 105, 136805 (2010).

[21] S. W. Han, H. Kwon, S. K. Kim, S. Ryu, W. S. Yun, D. H. Kim, J. H. Hwang, J.-S. Kang, J. Baik, H. J. Shin, and S. C. Hong, Phys. Rev. B 84, 045409 (2011).
[22] J. K. Ellis, M. J. Lucero, and G. E. Scuseria, Appl. Phys. Lett. 99, 261908 (2011).

[23] S. Horzum, H. Sahin, S. Cahangirov, P. Cudazzo, A. Rubio, T. Serin, and F. M. Peeters, Phys. Rev. B 87, 125415 (2013).

[24] H. Sahin, S. Tongay, S. Horzum, W. Fan, J. Zhou, J. Li, J. Wu, and F. M. Peeters, Phys. Rev. B 87, 165409 (2013).

[25] M. Kertesz and R. Hoffmann, J. Am. Chem. Soc. 106, 3453 (1984).

[26] K. Friemelt, M.-Ch. Lux-Steiner, and E. Bucher, J. Appl. Phys. 74, 5266 (1993).

[27] C. H. Ho, P. C. Liao, Y. S. Huang, T. R. Yang, and K. K. Tiong, J. Appl. Phys. 81, 6380 (1997).

[28] K. Friemelt, L. Kulikova, L. Kulyuk, A. Siminel, E. Arushanov, Ch. Kloc, and E. Bucher, J. Appl. Phys. 79, 9268 (1996).

[29] S. Tongay, H. Sahin, C. Ko, A. Luce, W. Fan, K. Liu, J. Zhou, Y.-S. Huang, C.-H. Ho, J. Yan, D. F. Ogletree, S. Aloni, J. Ji, S. Li, J. Li, F. M. Peeters, and J. Wu, Nat. Commun. 5, 3252 (2014).

[30] Y.-H. Lee, X.-Q. Zhang, W. Zhang, M.-T. Chang, C.-T. Lin, K.-D. Chang, Y.-C. Yu, J. T.-W. Wang, C.-S. Chang, L.-J. Li, and T.-W. Lin, Adv. Mater. 24, 2320 (2012).

[31] K.-K. Liu, W. Zhang, Y.-H. Lee, Y.-C. Lin, M.-T. Chang, C.-Y. Su, C.-S. Chang, H. Li, Y. Shi, H. Zhang, C.-S. Lai, and L.-J. Li, Nano Lett. 12, 1538 (2012).

[32] F. Banhart, J. Kotakoski, and A. V. Krasheninnikov, ACS Nano 5, 26 (2011).

[33] G. Kresse and J. Hafner, Phys. Rev. B 47, 558 (1993).

[34] G. Kresse and J. Furthmuller, Phys. Rev. B 54, 11169 (1996).

[35] J. P. Perdew, K. Burke, and M. Ernzerhof, Phys. Rev. Lett. 77, 3865 (1996).

[36] G. Kresse and D. Joubert, Phys. Rev. B 59, 1758 (1999).

[37] H. J. Monkhorst and J. D. Pack, Phys. Rev. B 13, 5188 (1976).

[38] S. Tongay, J. Suh, C. Ataca, F. Wen, A. Luce, J. S. Kang, J. Liu, C. Ko, J. Zhou, J. Li, J. C. Grossman, and J. Wu, Sci. Rep. 3, 2657 (2013).

[39] C. H. Ho, Y. S. Huang, K. K. Tiong, and P. C. Liao, J. Phys.: Condens. Matter 11, 5367 (1999).

[40] J. Zhang, J. M. Soon, K. P. Loh, J. H. Yin, J. Ding, M. B. Sullivian, and P. Wu, Nano Lett. 7, 2370 (2007). 